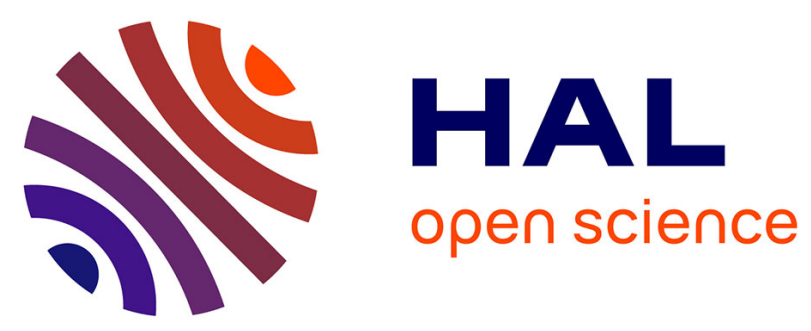

\title{
Protocol Adaptation of Optical Coherence Tomography in Lower Limb Arteries Revascularization
}

Maxime Dubosq, Benjamin O. Patterson, Richard Azzaoui, Thomas Mesnard, Agathe de Préville, Jonathan Sobocinski

\section{- To cite this version:}

Maxime Dubosq, Benjamin O. Patterson, Richard Azzaoui, Thomas Mesnard, Agathe de Préville, et al.. Protocol Adaptation of Optical Coherence Tomography in Lower Limb Arteries Revascularization. Annals of Vascular Surgery, 2019, 57, pp.257 - 260. 10.1016/j.avsg.2018.09.031 . hal-03486076

\section{HAL Id: hal-03486076 https://hal.science/hal-03486076}

Submitted on 20 Dec 2021

HAL is a multi-disciplinary open access archive for the deposit and dissemination of scientific research documents, whether they are published or not. The documents may come from teaching and research institutions in France or abroad, or from public or private research centers.
L'archive ouverte pluridisciplinaire HAL, est destinée au dépôt et à la diffusion de documents scientifiques de niveau recherche, publiés ou non, émanant des établissements d'enseignement et de recherche français ou étrangers, des laboratoires publics ou privés.

\section{(ㄷ)(1) $\$$}

Distributed under a Creative Commons Attribution - NonCommerciall 4.0 International 


\section{Protocol adaptation of OCT in lower limb arteries revascularisation}

2 M Dubosq ${ }^{1}$, BO Patterson ${ }^{2}$, R Azzaoui $^{1}$, T Mesnard $^{1,3}$, A De Préville ${ }^{1}$, J Sobocinski ${ }^{1,3}$

3

$4 \quad{ }^{1}$ Vascular surgery, Aortic centre, CHU Lille, France

$5 \quad{ }^{2}$ St George's Vascular Institute, NHS Trust, London

$6 \quad{ }^{3}$ Inserm U1008, University of Lille, France

7

8 Conflicts of interest:

9 J Sobocinski received speaker fees from AbbottVascular

11 Abstract

12 In lower limb arteries, assessment of stent apposition and/or the single opening of the 13 diseased artery remains poor since this relies on 2D angiogram. Extrapolating experience 14 gained with coronary arteries, optical coherence tomography (OCT) could provide 3D 15 reconstructions of the arterial wall and the stent implanted. A modified protocol of OCT 16 acquisition, which usually includes large amount of iodine contrast flush, is presented here in 173 patients with long and complex occlusion of the superficial femoral artery (SFA). Its 18 potential benefits and wider application to improve patient outcomes are discussed.

21 Introduction:

22 2D angiogram is used to assess intraoperative results of balloon angioplasty and/or stenting.

23 It is probably an acceptable modality in short lesions but is not able to provide 3D 24 appreciation of long complex arterial lesions. This could contribute toward some early \& 
mid-term procedural failures ${ }^{1}$. IVUS provides accurate intravascular evaluation of inadequate stent positioning or significant recoil missed with $2 \mathrm{D}$ angiogram ${ }^{2,3,4}$. Its use has not been widespread so far mainly due to its unproven cost-effectiveness ${ }^{4}$.

Optical Coherence Tomography is another endovascular imaging technique initially used in coronary arteries ${ }^{5}$. OCT is a recent technology for cross-sectional and 3D-imaging in biological systems with ultra-high resolution approaching that of histology. It provides endoluminal images and controls spatial opening of the stenotic/occluded artery after treatment. This lightening technology is based on echo lead-time measure and the intensity of a coherent light source in the near-infrared region instead of acoustic waves in IVUS ${ }^{4,6}$. Study comparison (to IVUS) in popliteal and infrapopliteal vessels showed OCT provided an excellent image quality and better visualisation of arterial wall layers of the different plaque components $^{3}$. In coronary arteries, OCT seems even better in detecting stent malapposition and edge dissections, but similar to IVUS in the assessment of lumen and stent dimensions ${ }^{5}$.

Two major limitations are usually described for arterial OCT in other situations: vessel diameter $>5 \mathrm{~mm}^{4} \&$ the need for iodine contrast injection to flush the artery for each OCT acquisition $^{4,7}$.

Arterial lesions in lower limb may be up to $50 \mathrm{~cm}$ long and maximum current length of OCT probes available in one imaging run is $7.5 \mathrm{~cm}$, meaning the single final control would require an unacceptable amount of iodine contrast.

We proposed and validated an adaptation of the OCT protocol based on isotonic saline in 3 patients treated for critical limb ischemia with complex superficial femoral artery (SFA) lesions. 
Three patients presented with TASC $C(n=1)$ and $D(n=2)$ lesions of the SFA were treated at Lille University Hospital on March 2018. Procedures were performed with flat-panel mobile detector (Elite, GE Healthcare). Parameters of the imaging system were configured to achieve a compromise between imaging quality and low radiation exposure. All procedures were performed under local anaesthesia with sedation. Controlateral puncture of the common femoral artery is our preferred option for SFA lesions. After placement of a 6Fr by $55 \mathrm{~mm}$ sheath (Flexor Ansel-1, CookMedical) with US guidance, patients received 50UI/kg of heparin. Initial 2D cine fluroscopic run confirmed the lesions depicted with preoperative US and/or CTA. All lesions were successfully crossed using either .018" СTO wire in 2 or using a subintimal approach with $.035^{\prime \prime}$ stiff hydrophilic guidewire in 1. Prolonged (>3minutes) and progressive balloon angioplasties (diameter $5 \mathrm{~mm}$ ) at 8 atm were performed in all 3 patients. 2D-cine fluoroscopy and OCT acquisitions were then executed to evaluate the need of reballooning or stent implantation.

OCT acquisitions require the use of OCT catheter (DragonFly Optis, AbbottVascular) and dedicated generator (Ilumien, AbbottVascular).

The protocol of OCT acquisitions comprised a first step where the shaft of the catheter was flushed with $2 \mathrm{cc}$ of iodine contrast and then a second step where the treated segment of artery was flushed via an automatic power injection of $80 \mathrm{cc}$ of isotonic saline at $10 \mathrm{cc} / \mathrm{sec}$ through the long sheath positioned just above the lesion to be evaluated. The OCT catheter was previously positioned at the level of the arterial segment being evaluated; it automatically detected the saline bolus arrival and launch the acquisition over a maximum length of $7.5 \mathrm{~cm}$ segment. In both control imaging modalities further stenting was required in all patients due to significant recoil and focal occlusive dissection. 
72 A $6 \mathrm{~mm}$ by $100 \mathrm{~mm}$ ABSOLUTE stent (AbbottVascular) was implanted in patient with TASC C

73 lesion; 1 patient with TASC D lesions received a $5 \mathrm{~mm}$ by $200 \mathrm{~mm}$ SUPERA stents 74 (AbbottVascular) +2 ABSOLUTE stents $6 \mathrm{~mm}$ by $150 \mathrm{~mm}$ proximally while the last patient

received 2 ABSOLUTE stents: $5 \mathrm{~mm}-150 \mathrm{~mm}$ and $6 \mathrm{~mm}-100 \mathrm{~mm}$ for long F1-F2 lesion. Postimplantation balloon inflation of the ABSOLUTE stent (AbbottVascular) was systematically performed but not for the SUPERA stent.

Final 2D cine fluoroscopy showed a good result with appropriate stent expansions (images) in all cases.

New OCT acquisitions showed discrepancies in stenting results in 2 patients:

In the TASC C patient, appearance of stent malapposition of the ABSOLUTE stent at some points and significant stenosis of the native proximal SFA that was thought to be free of lesions were depicted. Additional stent implantation after balloon angioplasty of the native proximal SFA and re-ballooning of the ABSOLUTE stent with a $6 \mathrm{~mm}$ balloon were performed [Figure 1].

The one TASC D patient required re-ballooning of the ABSOLUTE stents [Figure 2].

Median amount of isotonic saline volume injected per patient for OCT acquisitions was $400 \mathrm{cc}$ [400 to $480 \mathrm{cc}$ ]. Median lodine contrast (Xenetix $300 \mathrm{mg} / \mathrm{mL}$, Guerbet, France) volume injected was $27 c c$ [20cc to $40 \mathrm{cc}$ ]; median Dose Area Product was $14 \mathrm{mGycm}^{2}$ [8-21]. Procedural time was $115 \mathrm{~min}$ [85 to $140 \mathrm{~min}$ ].

The postoperative course was uneventful for all patients. Postoperative US duplex showed excellent flow restoration within the femoro-popliteal artery in all 3 patients. Patients were discharged under dual antiplatelet therapy for at least 2 months. 
97 segment.

Discussion:

The "gold standard" imaging control modality after endovascular revascularisation is 2Dangiography. Despite poor image resolution, 2D-angiography remains easy to use at low cost. The benefit of OCT technique in this setting would be to improve the accuracy and the quality of the initial revascularisation, since it visualises and characterizes the wall of the artery, visualising not only atherosclerotic plaque characteristics after stent implantation but also late restenosis phenomena ${ }^{6}$. Applications in the coronary arteries are well established

107 but few authors reported its use in the lower limb $b^{5,8}$.

108 (Frequency-Domain)-OCT, used in the 3 patients described, is able to detect intimal tears 109 and dissections after infra-inguinal angioplasty, which appears crucial for high-quality 110 imaging in long segments of larger arteries, such as femoral arteries. Moreover, super-fast 111 image acquisition helps to reduce the lead-time and flushing bolus required for blood replacement. Most of OCT acquisitions in the artery are frequency-domain based; reducing

113 the noise and thus the definition of images provided.

114 OCT requires intra-arterial injection of contrast agent to flush and clean the arterial lumen 115 during data acquisition. This represents a huge limitation when the arterial segment treated 116 is long. The use of Dextran and isotonic saline has already been proposed as an alternative to 117 contrast agent to reduce nephrotoxicity ${ }^{8}$, but no practical details or results on their 118 applicability and feasibility in clinical practice have been published so far.

119 Two main elements in the method here presented enable it to be achieved: 
120 First, the use of automatic power injection able to standardise the rate and volume injected,

121 then the placement of the long sheath close to the lesion and OCT probe avoiding flow

122 dilution and reducing the remaining blood streaks.

123 From our experience, the OCT acquisitions with hand-injected saline are not workable - the

124 flow-rate and volume injected are too low and not good enough to clean the arterial lumen

125 compatible with good OCT images quality.

126 By extension, the amount of saline volume injected for the whole procedure due to OCT

127 acquisitions could reach up to $1.5 \mathrm{~L}$ for long SFA lesions and should be taken into account in

128 patients with cardiac insufficiency - after discussion with the anaesthesiologist's team.

129 Moreover, in our practice, we only flush catheters and sheaths with isotonic heparinised

130 saline during procedure. In this specific setting, unprepared isotonic saline without

131 additional heparin was used for OCT acquisitions to avoid massive anticoagulation of the 132 patient that would expose them to an excessive bleeding risk.

133

134 In these first 3 patients, OCT depicted defects in stent expansion and de novo lesions not

135 seen with the initial 2D angiogram or the preoperative duplex scan. This tool potentially

136 helped to improve the outcomes of these patients by reducing the risk of postoperative

137 early thrombosis; this also represent a valuable tool in determining the need for additional

138 stenting and/or balloon angioplasty.

139 Although OCT is an expensive technique there is potential for preventing early postoperative

140 failure and subsequent re-intervention. The protocol proposed in this work based on the

141 injection of isotonic saline would extend its applicability to lower limb revascularisation. This

142 could be worthwhile assessing as part of a prospective randomized study on clinical 143 perspectives but also health economical aspects. 
1. Paraskevopoulos I, Spiliopoulos S, Davlouros P, Karnabatidis D, Katsanos K,

148 Alexopoulos D, et al. Evaluation of Below-the-Knee Drug-Eluting Stents With Frequency-

149 Domain Optical Coherence Tomography: Neointimal Hyperplasia and Neoatherosclerosis.

150 J Endovasc Ther 2013;20:80-93

151 2. Karnabatidis D, Katsanos K, Paraskevopoulos I, Diamantopoulos A, Spiliopoulos S, 152 Siablis D. et al Frequency-Domain Intravascular Optical Coherence Tomography of the 153 Femoropopliteal Artery. Cardiovasc Intervent Radiol. 2011;34:1172-1181

154 3. Eberhardt KM, Treitl M, Boesenecker K, Maxien D, Reiser M, Rieger J. Prospective 155 Evaluation of Optical Coherence Tomography in Lower Limb Arteries Compared with 156 Intravascular Ultrasound. J Vasc Interv Radiol 2013; 24:1499-1508

157 4. Spiliopoulos S, Kitrou P, Katsanos K, Karnabatidis D. FD-OCT and IVUS intravascular 158 imaging modalities in peripheral vasculature. Expert Review of Medical Devices, $159 \quad 2017 ; 14: 127-134$ 5. Fujino $Y$, Bezerra HG, Attizzani GF, Wang W, Yamamoto $H$, Chamié $D$, et al. Frequency-domain optical coherence tomography assessment of unprotected left main coronary artery disease-a comparison with intravascular ultrasound. Catheter Cardiovasc Interv. 2013;82:E173-83

6. Meissner OA, Rieber J, Babaryka G, Oswald M, Reim S, Siebert U, et al. Intravascular

165 Optical Coherence Tomography: Comparison with Histopathology in Atherosclerotic 166 Peripheral Artery Specimens. J Vasc Interv Radiol 2006; 17:343-349

167 7. Farooq MU, Khasnis A, Majid A, Kassab MY. The role of optical coherence tomography in vascular medicine. Vascular Medicine 2009; 14: 63-71 
169 8. Ozaki Y, Kitabata H, Tsujioka H, Hosokawa S, Kashiwagi M, Ishibashi K, et al. 170 Comparison of contrast media and low-molecular-weight dextran for frequency-domain 171 optical coherence tomography Circ J. 2012;76:922-7

172 9. Schwindt AG, et al. Lower Extremity Revascularization Using Optical Coherence 173 Tomography-Guided Directional Atherectomy: Final Results of the EValuatlon of the 174 PantheriS Optlcal COherence Tomography ImagiNg Atherectomy System for Use in the 175 Peripheral Vasculature (VISION) Study J Endovasc Ther. 2017;24:355-366 
178 Figures legend

179

180 Figure 1: patient with TASC C lesion; control OCT after initial stenting showed malapposition

181 of the laser-cut stent and stenosis of the proximal native artery (centre) missed with the 2D

182 cine fluoroscopic run (left). The final angiogram was satisfying (right).

183 Figure 2: patient with TASC D lesion; the distal part of the SFA received a woven self-

184 expansible stent (left). The final OCT control before (left) and after (centre and right) a

185 second prolonged balloon angioplasty. 


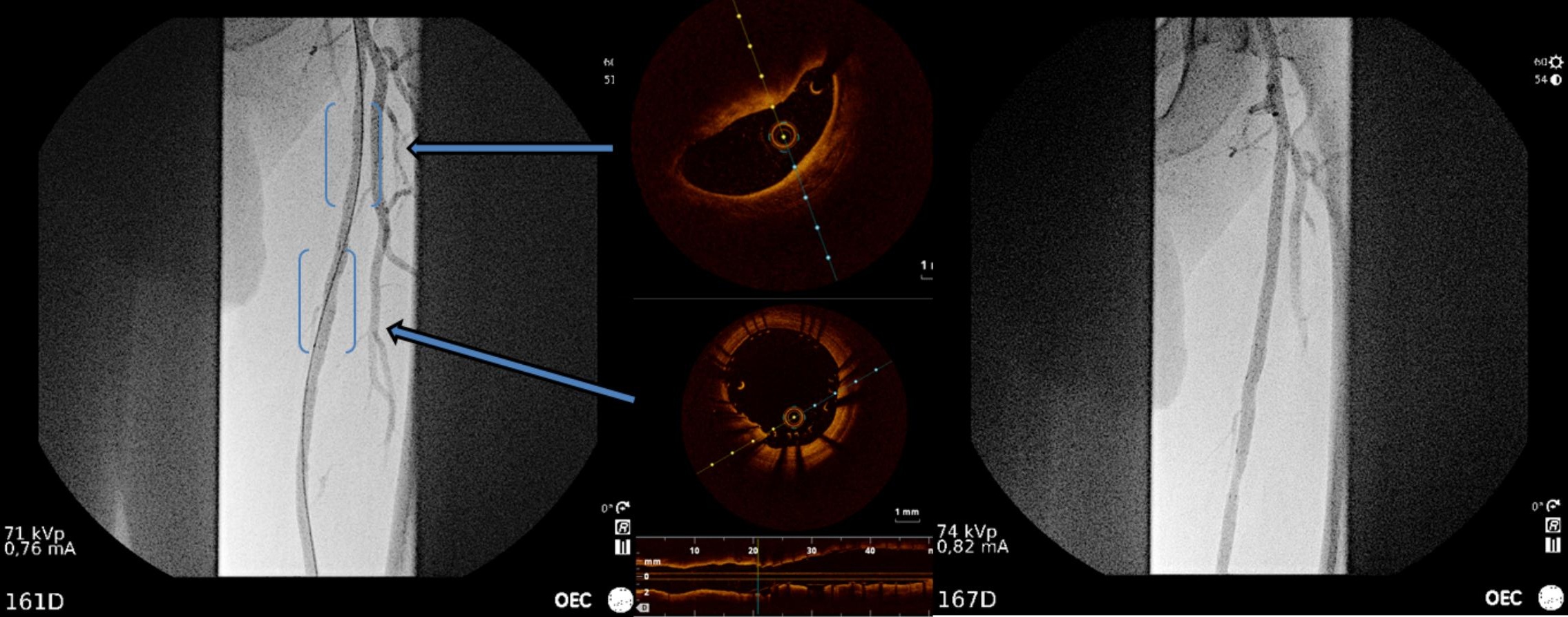



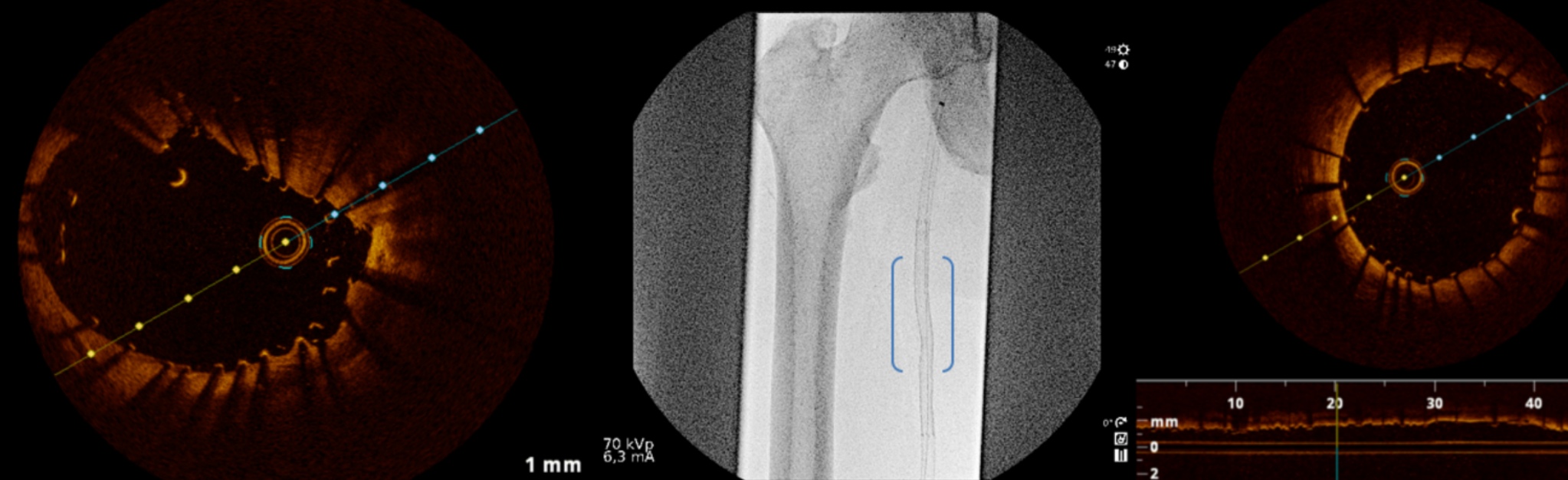

$1 \mathrm{~m}$

典

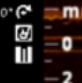

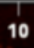

40 $x+x+0,5$ 